\title{
Increased substantia nigra echogenicity correlated with visual hallucinations in Parkinson's disease: a Chinese population-based study
}

\author{
Ting $\mathrm{Li}^{1} \cdot$ Jing Shi ${ }^{1} \cdot$ Bin Qin ${ }^{2} \cdot$ Dongsheng $\mathrm{Fan}^{3} \cdot \mathrm{Na} \mathrm{Liu}^{3} \cdot$ Jingnian $\mathrm{Ni}^{1} \cdot$ Tianqing Zhang ${ }^{1} \cdot \mathrm{Hufang} \mathrm{Zhou}^{1} \cdot$ \\ Xiaoqing $\mathrm{Xu}^{1} \cdot$ Mingqing Wei ${ }^{1} \cdot$ Xuekai Zhang $^{1} \cdot$ Xiangzhu Wang $^{1} \cdot$ Jianping Liu ${ }^{4} \cdot$ Yongyan Wang ${ }^{5} \cdot$ Jinzhou Tian $^{1}$
}

Received: 14 February 2019 / Accepted: 15 October 2019 / Published online: 22 November 2019

(C) The Author(s) 2019

\begin{abstract}
As a noninvasive technique, transcranial sonography (TCS) of substantia nigra (SN) has gradually showed its effectiveness not only in diagnosis but also in understanding clinical features of Parkinson's Disease (PD). This study aimed to further evaluate TCS for clinical diagnosis of PD, and to explore the association between sonographic manifestations and visual hallucinations (VH). A total of 226 subjects including 141 PD patients and 85 controls were recruited. All participants received TCS. A series of rating scales to evaluate motor and non-motor symptoms were performed in PD patients. Results showed that 172 subjects were successfully assessed by TCS. The area of SN was greater in PD patients than that in controls $(P<0.001)$. As receiver-operating characteristic (ROC) curve analysis showed, the best cutoff value for the larger SN echogenicity size was $23.5 \mathrm{~mm}^{2}$ (sensitivity $70.3 \%$, specificity $77.0 \%)$. Patients with VH had larger SN area $(P=0.019)$, as well as higher Non-Motor Symptoms Scale (NMSS) scores $(P=0.018)$. Moreover, binary logistic regression analysis indicated that $\mathrm{SN}$ hyperechogenicity (odds ratio = 4.227, $P=0.012$ ) and NMSS scores (odds ratio $=0.027, P=0.042$ ) could be the independent predictors for VH. In conclusion, TCS can be used as an auxiliary diagnostic tool for Parkinson's disease. Increased SN echogenicity is correlated with VH in Parkinson's disease, possibly because the brain stem is involved in the mechanism in the onset of VH. Further studies are needed to confirm these findings.
\end{abstract}

Keywords Parkinson's disease $\cdot$ Transcranial sonography $\cdot$ Visual hallucinations $\cdot$ Clinical features $\cdot$ Diagnosis

\section{Introduction}

Parkinson's disease (PD) is one of the most common neurodegenerative diseases. Though dopamine transporter positron emission computed tomography (DAT-PET) is proved to be

Ting Li and Jing Shi contributed equally to this work.

Jinzhou Tian

jztian@hotmail.com

1 The Neurology Center, Dongzhimen Hospital, Beijing University of Chinese Medicine, Beijing 100700, China

2 Beijing Hospital, Beijing 100730, China

3 Peking University Third Hospital, Beijing 100191, China

4 Center for Evidence-Based Chinese Medicine, Beijing University of Chinese Medicine, Beijing 100029, China

5 Institute of Clinical Medicine, China Academy of Chinese Medical Sciences, Beijing 100700, China an effective diagnostic technique [1], it has not been widely used due to high expense and radio action. The diagnosis of PD mainly relies on clinical manifestations [2]. As a noninvasive technique, transcranial sonography (TCS) is potentially useful for the diagnosis of PD by showing the structural changes in substantia nigra (SN). Even though previous studies have proved that the specificity was $88.2-85 \%$ and the sensitivity was $84-94.9 \%$ in diagnostic accuracy of TCS in PD patients [3, 4], and the concordance rate between TCS patterns and PD diagnosis increased from 87 to $95 \%$ in a 4year follow-up [5]. Still, the data based on Chinese population need to be supplemented.

It is still unclear whether the extent of $\mathrm{SN}$ hyperechogenicity correlates with only motor symptoms or other clinical status, more precise quantification of the damage is required in order to improve extensive and in-depth understanding in PD. There were studies exploring the correlation between SN echogenicity and clinical features, and found that patients with larger hyperechogenic SN area tended to have 
severer motor and non-motor symptoms [6-8]; however, visual hallucinations $(\mathrm{VH})$, one of the most common psychotic symptoms, which was reported to affect 9.8 to $82.7 \%$ of PD population in different stages [9-11], in relation to $\mathrm{SN}$, have not been reported before.

Our objective is to evaluate the validity of TCS for the diagnosis of PD in Chinese population, and to investigate the correlation of sonographic manifestations with clinical features, especially in VH.

\section{Subjects and methods}

\section{Subjects}

From May 2015 to March 2018, 141 participants with PD were recruited after giving their informed consent at the Department of Neurology, Dongzhimen Hospital. These 141 participants all met the criteria of the UK Parkinson's Disease Society Brain Bank [2], and had enough audio-visual functions to complete motor and non-motor symptom evaluation test. Participants were excluded if they had possible dementia with Lewy bodies (DLB) according to 2005 DLB diagnostic criteria (DLBC-3) [12]. From May 2015 to January 2018, 85 volunteers without parkinsonism consented to participate as control subjects from the Department of Neurology, Dongzhimen Hospital and Poster recruitment. All volunteers were assessed by neurology specialists and were excluded if they had positive family history of PD or the possibility of parkinsonism.

This study had been approved by the ethics committee of Dongzhimen Hospital, the First Affiliated Hospital of Beijing University of Chinese Medicine.

\section{Transcranial sonography operation and diagnosis standard}

Through the preauricular acoustic bone window, a qualified operator, who was blind to the clinical information of the subjects, examined the echogenicity of the SN using a $1.82 \mathrm{MHz}$ sonographic device (ACUSON Anteres, SIEMENS) with a depth of $15-18 \mathrm{~cm}$ and a dynamic range of $26 \mathrm{~dB}$. The SN was scanned through both temporal bone windows in the axial plane. After identifying the butterflyshaped hypoechogenic midbrain surrounded by the hyperechogenic of basal cistern, the clearest image of the hyperechogenic signal in the SN region was stored (Fig. 1). The areas of SN hyperechogenicity in the midbrain were measured manually by the same operator.

The SN hyperechogenicity were obtained from right and left temporal windows. Some of the patients were measured from only one temporal window if it was impossible to obtain images from both sides. The larger $\mathrm{SN}$ echogenic area $\left(\mathrm{SN}_{\mathrm{L}}\right)$ was used to perform receiver-operating characteristic (ROC) curve analysis.

\section{Clinical features assessment}

We recorded the clinical parameters including age, gender, disease duration, and symptoms of onset. To assess the disease severity, the PD patients received evaluation of the Unified Parkinson's Disease Rating Scale (UPDRS) [13], and their Hoehn and Yahr Stage (H-Y stage) was graded during the 12-h medication "off" phase. Participants received a series of tests to evaluate their non-motor symptoms (NMS). The Non-Motor Symptoms Scale (NMSS) [14] was used to test the overall status. The PDSS [15], CSI [16], and PFS [17] were used to evaluate the participants' sleep disorder, constipation, and fatigue symptom respectively. Mini-Mental State Examination (MMSE) [18] was used to evaluate the cognitive function. The degree of depression and anxiety was assessed by Hamilton Depression Scale (HAMD) [19] and Hamilton Anxiety Scale (HAMA) [20]. And the living quality was evaluated by PDQ-39 [21]. The presence of VH was defined according to item 13 from NMSS scale.

\section{Statistical analysis}

The data were analyzed by using SPSS 22.0. The descriptive statistics were given as mean value \pm standard deviation. The categorical statistics were recorded as count and percentage data. Descriptive statistics received normality test, and further variation analysis was performed by two-sample $t$ test and Mann-Whitney $U$ test. The statistical difference of categorical data was calculated by chi-square test. Correlations of the scale scores and $\mathrm{SN}_{\mathrm{L}}$ were performed by Pearson correlation coefficients. Statistical significance was set at $P<0.05$. The ROC curve analysis was applied to acquire the cutoff value to distinguish PD patients from normal controls. The classifier for ROC curve analysis was defined as The UK Brain Bank Criteria [2]. For each point in the curve, sensitivity and 1specificity were shown for a certain cutoff value. And the best cutoff value was defined as where the sum of sensitivity and specificity was highest.

A binary logistic regression analysis was used to determine the most significant variables which were independently correlated with $\mathrm{VH}$. As a dependent variable, the presence of $\mathrm{VH}$ was defined as a binary variable. Age, duration, $\mathrm{SN}_{\mathrm{L}}$, UPDRS total scores, NMSS scores, and MMSE scores were included as covariates.

\section{Results}

Among the 226 subjects, TCS was successfully performed in 172 subjects $(76.10 \%, 111$ PD patients, 72 men and 39 
Fig. 1 Sonographic images of the mesencephalic brainstem in a healthy control (a) and a patient with Parkinson's disease (b). The butterfly-shaped mesencephalic brainstem was surrounded by the hyperechogenic basal cisterns The patient with Parkinson's disease exhibited hyperechogenic signals encircled by lines at both sides of SN, which were not seen in the control

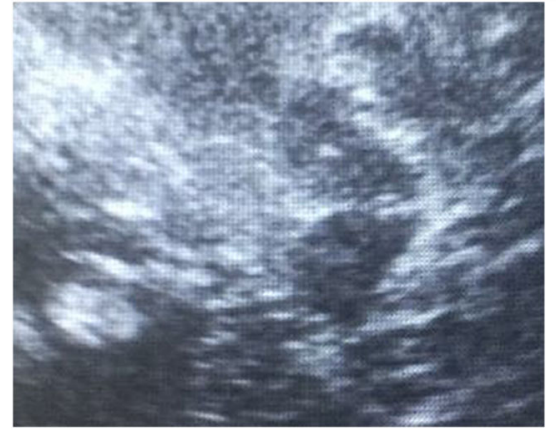

(a)

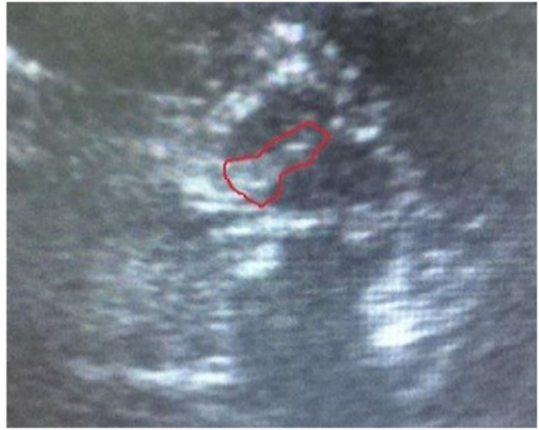

(b) women, and 61 normal controls, 38 men and 23 women). Fifty-four subjects $(23.90 \%)$ failed to acquire sonographic image due to poor penetration of ultrasound through both bony windows, and were excluded from further analysis.

\section{SN echogenicity in the participants}

Table 1 shows the basic characteristics, scale evaluations, and SN echogenicity data among PD and control. There was no difference in gender and age between two groups. For PD patients, the mean SN hyperechogenic area was $21.61 \pm 18.00 \mathrm{~mm}^{2}$ on the right side and $25.44 \pm 20.24 \mathrm{~mm}^{2}$ on the left. For normal controls, the mean SN hyperechogenic area was $8.28 \pm 14.58$

Table 1 Data of SN in the normal controls and PD patients

\begin{tabular}{llll}
\hline & $\begin{array}{l}\text { PD patients } \\
(N=111)\end{array}$ & $\begin{array}{l}\text { Controls } \\
(N=61)\end{array}$ & $P$ value \\
\hline Age (years) & $66.35 \pm 9.10$ & $63.10 \pm 11.44$ & 0.059 \\
Sex, male,$n(\%)$ & $72(64.86 \%)$ & $38(62.30 \%)$ & 0.737 \\
SN-right $\left(\mathrm{mm}^{2}\right)$ & $21.61 \pm 18.00$ & $8.28 \pm 14.58$ & $0.000^{*}$ \\
SN-left $\left(\mathrm{mm}^{2}\right)$ & $25.44 \pm 20.24$ & $12.44 \pm 15.97$ & $0.000^{*}$ \\
SN $\left(\mathrm{mm}^{2}\right)$ & $32.30 \pm 18.42$ & $14.61 \pm 17.55$ & $0.000^{*}$ \\
UPDRS & $34.33 \pm 12.65$ & $/$ & $/$ \\
NMSS & $49.73 \pm 23.16$ & $/$ & $/$ \\
MMSE & $27.28 \pm 3.98$ & $/$ & $/$ \\
PDSS & $115.21 \pm 19.88$ & $/$ & $/$ \\
CSI & $21.69 \pm 11.67$ & $/$ & $/$ \\
PFS & $47.58 \pm 11.01$ & $/$ & $/$ \\
HAMA & $11.26 \pm 6.35$ & $/$ & $/$ \\
HAMD & $8.68 \pm 4.34$ & $/$ & $/$ \\
PDQ-39 & $32.52 \pm 21.25$ & $/$ & $/$ \\
\end{tabular}

$S N$ substantia nigra, $S N$-right right $\mathrm{SN}$ echogenic area, $S N$-left left $\mathrm{SN}$ echogenic area, $S N_{L}$ the larger $\mathrm{SN}$ echogenic area, UPDRS Unified Parkinson's Disease Rating Scale, NMSS The Non-Motor Symptoms Scale, PDSS The Parkinson's Disease Sleep Scale, CSI The Constipation Severity Instrument, PFS Parkinson Fatigue Scale, HAMA Hamilton Anxiety Scale, HAMD Hamilton Depression Scale, $P D Q-3939$ item Parkinson's Disease Questionnaire

$* P<0.05$ $\mathrm{mm}^{2}$ on the right side and $12.44 \pm 15.97 \mathrm{~mm}^{2}$ on the left, both were statistically smaller than $\mathrm{PD}$ group $(P<0.001$, right side; $P$ $<0.001$, left side). The mean size of $\mathrm{SN}_{\mathrm{L}}$ in $\mathrm{PD}$ group was 32.30 $\pm 18.42 \mathrm{~mm}^{2}$, which was significantly greater than the control group $\left(14.61 \pm 17.55 \mathrm{~mm}^{2}, P<0.001\right)$.

\section{Discriminative power of SN echogenicity for PD}

A ROC curve for the discrimination of PD patients and control subjects is shown in Fig 2. The larger $\mathrm{SN}$ echogenic areas $\left(\mathrm{SN}_{\mathrm{L}}\right)$ were taken to plot the ROC curve. The ideal diagnostic threshold should yield the highest sum of sensitivity and specificity. Therefore, the point situated in the top left corner of the curve would be the best diagnostic cutoff value. We marked the point in

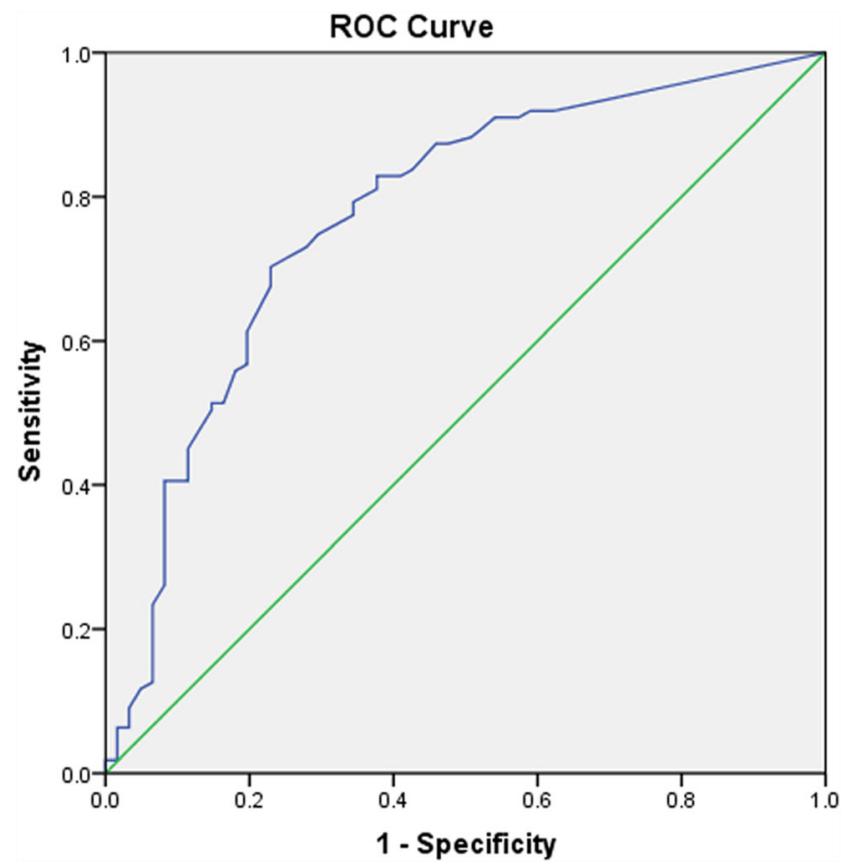

Fig. 2 ROC curve for the differentiation of PD vs. control subjects. The larger $\mathrm{SN}$ echogenic areas $\left(\mathrm{SN}_{\mathrm{L}}\right)$ were taken for the plotting of the ROC curve in the entire cohort. Asterisk marks the point in the SNL curve for the cutoff of $23.5 \mathrm{~mm}^{2}$ (area under the curve $=0.775$ ). The sensitivity value was $70.3 \%$, and the specificity value was $77.0 \%$ 
the $\mathrm{SN}_{\mathrm{L}}$ curve for the cutoff value of $23.5 \mathrm{~mm}^{2}$. At this point, the sensitivity was $70.3 \%$, and the specificity was $77.0 \%$. The area under the curve was 0.775 . Based on this cutoff value, $78 \mathrm{PD}$ patients $(70.27 \%)$ were classified $\mathrm{SN}_{\mathrm{L}} \geq 23.5 \mathrm{~mm}^{2} ; 14$ control subjects $(22.95 \%)$ were classified $\mathrm{SN}_{\mathrm{L}} \geq 23.5 \mathrm{~mm}^{2}$.

\section{Correlation between clinical features and SN echogenicity}

We analyzed the correlation between age, H-Y stage, disease duration, UPDRS scores, UPDRS sub domain scores, NMSS scores, PDSS scores, CSI scores, PFS scores, HAMA scores, HAMD scores, MMSE scores, PDQ-39 scores, and $\mathrm{SN}_{\mathrm{L}}$ with the Pearson correlation analysis. The UPDRS-II scores were significantly correlated with $\mathrm{SN}_{\mathrm{L}}(r=0.196, P=0.039)$. Other scales did not show the correlation.

\section{Correlation of variables in PD with VH}

In PD group, 18 (16.2\%) of 111 patients had VH, including 4 possible dementia associated with Parkinson's disease (PDD) and 14 cases with normal cognitive function [22]. We found that sonographic image and clinical features had special connection with $\mathrm{VH}$; thus, we grouped the 111 patients according to with or without $\mathrm{VH}$. As Table 2 shows, the mean age of $\mathrm{VH}$ group was much older than the non-VH group, and the difference had statistical significance $(P=0.008)$. The mean area of $\mathrm{SN}_{\mathrm{L}}$ in $\mathrm{PD}$ patients with $\mathrm{VH}$ was larger than patients without $\mathrm{VH}$ in statistics $(P=0.018)$. The NMSS and UPDRS-II scores had statistical significance ( $P=0.027$, NMSS; $P=0.036$, UPDRS-II) between $\mathrm{VH}$ group and non-VH group. The $\mathrm{VH}$ was not significantly associated with UPDRS total (UPDRS-T) and MMSE scores, although VH group had a higher level in UPDRS-T and a lower level in MMSE.

Further binary logistic regression analysis was used to identify the correlation between $\mathrm{VH}$ and other clinical features. Among age, duration, $\mathrm{SN}_{\mathrm{L}}$, UPDRS total scores, NMSS scores and MMSE scores, $\mathrm{SN}_{\mathrm{L}}$ and NMSS scores were the only two variables included in the final model. This demonstrated that there was a significant correlation between the $\mathrm{VH}$ and $\mathrm{SN}_{\mathrm{L}}$ (odds ratio $=4.227, P=0.012$ ) and NMSS total scores (odds ratio $=0.027, P$ $=0.042$ ).

\section{Discussion}

This study explored the correlation between SN echogenicity and clinical characteristics in Chinese PD patients. We found that the SN echogenicity area in PD patients with $\mathrm{VH}$ was significantly higher than those without $\mathrm{VH}$, which to our knowledge, had not been reported before.

As the results showed, 18 (16.2\%) of 111 PD patients had VH, similar to another study in China (14.06\%) [23]. Previous studies demonstrated that the impairment of visual input and central visual processing, as well as the impairment of brainstem regulation of the sleep-wake cycle may be the possible mechanisms $[24,25]$. The exact pathogenesis of VH in PD patients is not clearly understood. Current studies concerning VH in PD and neuroimaging mainly used the technology of functional magnetic resonance imaging (fMRI), positron emission tomography (PET), and single photon emission computerized tomography (SPECT). Based on imaging studies, there was evidence which

Table 2 Clinical data of PD patients grouped according to VH

\begin{tabular}{|c|c|c|c|c|}
\hline & $\begin{array}{l}\text { Total } \\
(n=111)\end{array}$ & $\begin{array}{l}\text { With visual hallucination } \\
(n=18)\end{array}$ & $\begin{array}{l}\text { Without visual hallucination } \\
(n=93)\end{array}$ & $P$ value \\
\hline Age (years) & $66.35 \pm 9.10$ & $70.67 \pm 8.85$ & $65.52 \pm 8.96$ & $0.008^{*}$ \\
\hline Sex , male , $n(\%)$ & $72(64.86 \%)$ & $15(83.33 \%)$ & $57(61.29 \%)$ & 0.073 \\
\hline SN-right & $21.61 \pm 18.00$ & $31.06 \pm 20.09$ & $19.74 \pm 17.06$ & $0.028 *$ \\
\hline SN-left & $25.44 \pm 20.24$ & $29.17 \pm 23.65$ & $24.72 \pm 19.58$ & 0.528 \\
\hline $\mathrm{SN}_{\mathrm{L}}$ & $32.30 \pm 18.42$ & $41.67 \pm 20.75$ & $30.48 \pm 17.48$ & $0.018 *$ \\
\hline UPDRS-I & $2.25 \pm 1.72$ & $2.94 \pm 2.29$ & $2.11 \pm 1.57$ & 0.201 \\
\hline UPDRS-II & $12.41 \pm 5.18$ & $14.22 \pm 5.56$ & $12.06 \pm 5.06$ & $0.036^{*}$ \\
\hline UPDRS-III & $18.20 \pm 6.77$ & $19.28 \pm 6.62$ & $17.99 \pm 6.82$ & 0.260 \\
\hline UPDRS-IV & $1.47 \pm 1.73$ & $1.22 \pm 1.48$ & $1.52 \pm 1.77$ & 0.344 \\
\hline UPDRS-Total & $34.33 \pm 12.65$ & $37.67 \pm 12.87$ & $33.69 \pm 12.57$ & 0.064 \\
\hline MMSE & $27.28 \pm 3.98$ & $26.56 \pm 3.52$ & $27.42 \pm 4.06$ & 0.121 \\
\hline NMSS & $49.73 \pm 23.16$ & $61.50 \pm 28.48$ & $47.42 \pm 21.40$ & $0.027 *$ \\
\hline Disease duration (years) & $5.97 \pm 5.57$ & $6.17 \pm 5.69$ & $5.94 \pm 5.58$ & 0.939 \\
\hline
\end{tabular}

$S N$ substantia nigra, $S N$-right right $\mathrm{SN}$ echogenic area, $S N$-left left $\mathrm{SN}$ echogenic area, $S N_{L}$ the larger SN echogenic area, UPDRS Unified Parkinson's Disease Rating Scale, MMSE Mini-Mental State Examination, NMSS The Non-Motor Symptoms Scale

$* P<0.05$ 
supported the hypothesis that abnormality and dysfunction in visual cortex and cholinergic structures such as the SN and pedunculopontine nucleus were to blame for VH [26]. However, the interaction between SN echogenicity and VH in PD was rarely investigated before.

Transcranial sonography can detect trace metal accumulation in deep brain structures with higher sensitivity than conventional MRI. In PD, particularly, the accumulation of iron has been suggested as an important substrate of extended SN echogenicity [27]. Berg reported close connections between SN echogenicity and elevated iron content of the SN in both animal and human studies [28, 29]. However, only a few cases of parkinsonism with VH have been reported with significant midbrain iron accumulation [30]. There was no direct evidence of correlation between iron accumulation in the SN and VH in PD. Another mechanism of enhanced SN echogenicity in PD might be microglial activation [31], which was demonstrated in midbrain specimens from postmortem PD patients [31, 32] and PD rat model [33]. A case report of $\mathrm{PD}$ with $\mathrm{VH}$ and delusions showed that neuronal loss with gliosis was noteworthy in the substantia nigra, locus ceruleus, dorsal vagal nucleus, nucleus basalis of Meynert, and intermediate lateral nuclei, and cholinergic projections from the nucleus basalis of Meynert could be responsible for generation of hallucinations and delusions [34]. However, there was also no direct evidence of correlation between microglial activation in the $\mathrm{SN}$ and $\mathrm{VH}$ in PD.

Among assessment of SN echogenicity, PD with VH had larger echogenic areas compared to those without. The correlation was further confirmed by binary logistic regression analysis. Together with these findings, we preliminarily inferred that the impairment of cholinergic structure in $\mathrm{SN}$ might contribute to VH in PD. Future studies may combine multiple imaging modalities to identify the main nerve damage in SN from iron accumulation, microglial activation, and other pathological processes.

Previous studies $[35,36]$ showed that VH in PD were associated with disease duration, dopamine agonist use, sleep quality, and cognition. In our study, there was significant difference between PD with VH and those without VH in age, NMSS, and UPDRS-II scores, while no statistic difference was found in disease duration, MMSE, and UPDRS-III scores. Moreover, there is no correlation between echogenicity and age, H-Y stage, NMSS scores, UPDRS-III, or disease duration. These results indicated that the correlation between VH and SN echogenicity was independent from NMSS scores, UPDRS-III scores, and disease duration. Considering there was no correlation between age and SN echogenicity in our study, similar conclusion was drew out in another Asian population-based study [8] (no difference in age between $\mathrm{SN} \geq 18 \mathrm{~mm}^{2}$ group and $\mathrm{SN}<18 \mathrm{~mm}^{2}$ group). We could initially speculate that the correlation between $\mathrm{VH}$ and $\mathrm{SN}$ echogenicity was also independent from age. However, PD with VH had higher UPDRS-II scores, and the UPDRS-II scores were associated with $\mathrm{SN}_{\mathrm{L}}$. Therefore, we can not currently rule out the impact of UPDRS-II on the result that PD with VH had larger echogenic area.

There have been some studies concerning the relationship between SN echogenicity and clinical features of PD. Results showed that depression, urinary incontinence, UPDRS-II, SCOPA-AUT, and postural instability gait difficulty were related to SN hyperechogenicity $[8,37]$. In our cohort, only UPDRS-II scores were associated with $\mathrm{SN}_{\mathrm{L}}$, consistent with the result of Zhou's study [8] based on Chinese patients. Yet, the underlying mechanism remains unclear. PD patients with VH had higher UPDRS-II scores might be a possible reason.

There has not been a well-accepted diagnostic value of hyperechogenic area to differentiate PD $[8,37]$. Our study applied ROC curve analysis to investigate the cutoff value for $\mathrm{SN}+$. The cutoff value in our study is higher compared with other researches $[8,38,39]$. To investigate the reason for this phenomenon, Table 3 demonstrates the clinical features of our study and 5 other studies [7, 8, 38-40]. The mean age was calculated using the information from all the participants, while the disease duration and H-Y stage were from only the PD patients. There seems to be a trend that as age and disease duration grow, the cutoff value also grows. Similar conclusion was carried out in a TCS hyperechogenicity area study [41] among healthy infants, children, as well as healthy adults. The result indicated an age-related increase of the hyperechogenic area of SN; thus, older age in our study might lead to an enlarged echogenic size level.

The TCS penetration rate was an important issue and might affect the study result to some extent, because some

Table 3 Clinical characteristic comparison among different studies

\begin{tabular}{lllll}
\hline & Cutoff value $\left(\mathrm{mm}^{2}\right)$ & Mean age (years) & Disease duration (years) & H-Y stage \\
\hline Our study & 23.5 & $64.85 \pm 11.23$ & $5.97 \pm 5.57$ & $2.46 \pm 0.70$ \\
Zhou H-Y [7] & 18 & $60.77 \pm 10.26$ & $5.59 \pm 4.21$ & $2.02 \pm 0.81$ \\
Berg D [28] & 19 & 63 & 6 & 2 \\
Kim J-Y [29] & 20 & $56.9 \pm 12.9$ & $3.5 \pm 3.2$ & 2.06 \\
Luo S-F [30] & 20 & $58.7 \pm 6.8$ & $5.06 \pm 3.11$ & - \\
Bartova P [6] & 25 & $68.59 \pm 10.37$ & $6.8 \pm 4.3$ & 2 \\
\hline
\end{tabular}


participants were excluded due to bad penetration of ultrasound through both bony windows. Among the 226 participants, 172 acquired adequate SN sonographic image; the insufficient penetration rate was $23.9 \%$ (21.3\% in PD group, $28.2 \%$ in control group). In studies among Caucasian population, the insufficient penetration rate was in a level of 6.9$15.5 \%$ [7, 42, 43], while several Asian studies reported a higher temporal insufficiency rate varied from 20.5 to $30.2 \%$ $[8,39]$. The Asian and Caucasian temporal bone structure diversity may cause the difference. Moreover, hyperostosis frontalis interna occurs as age grows, especially in female [44]. This might be another reason why the insufficient penetration rate was higher in our study.

In conclusion, enlarged $\mathrm{SN}$ hyperechogenic area significantly correlates with the presence of VH in a Chinese population with PD. This finding may provide evidence for brain stem involvement mechanism in the onset of VH. However, with a relatively small sample size of $\mathrm{VH}$ group, further studies are needed to confirm our findings.

Funding information This study was supported by the National Natural Science Foundation of China (NO.81473518 and No.81573824), Beijing Municipal Science and Technology Commission (No.Z141107002515019), and the Capital Health Research and Development of Special (No.SF2014-1-4191).

\section{Compliance with ethical standards}

Conflict of interest The authors declare that they have no conflict of interest.

Open Access This article is distributed under the terms of the Creative Commons Attribution 4.0 International License (http:// creativecommons.org/licenses/by/4.0/), which permits unrestricted use, distribution, and reproduction in any medium, provided you give appropriate credit to the original author(s) and the source, provide a link to the Creative Commons license, and indicate if changes were made.

\section{References}

1. Stoessl AJ (2012) Neuroimaging in the early diagnosis of neurodegenerative disease. Transl neurodegener 1:5

2. Lees AJ, Hardy J, Revesz T (2009), Parkinson's disease. Lancet (London, England) 373:2055-2066.

3. Stenc Bradvica I, Mihaljevic I, Butkovic-Soldo S, Kadojic D, Titlic M, Bradvica M, Kralik K (2015) Transcranial sonography and the pocket smell test in the differential diagnosis between Parkinson's disease and essential tremor. Neurol Sci 36:1403-1410

4. Tao A, Chen G, Deng Y, Xu R (2019) Accuracy of transcranial sonography of the substantia nigra for detection of Parkinson's disease: a systematic review and meta-analysis. Ultrasound Med Biol 45:628-641

5. Monaco D, Berg D, Thomas A, Di Stefano V, Barbone F, Vitale M, Ferrante C, Bonanni L et al (2018) The predictive power of transcranial sonography in movement disorders: a longitudinal cohort study. Neurol Sci 39:1887-1894

6. Yu SY, Cao CJ, Zuo LJ, Chen ZJ, Lian TH, Wang F, Hu Y, Piao YS et al (2018) Clinical features and dysfunctions of iron metabolism in
Parkinson disease patients with hyper echogenicity in substantia nigra: a cross-sectional study. BMC Neurol 18:9

7. Bartova P, Skoloudik D, Ressner P, Langova K, Herzig R, Kanovsky P (2010) Correlation between substantia nigra features detected by sonography and Parkinson disease symptoms. J Ultras Med 29:37-42

8. Zhou HY, Sun Q, Tan YY, Hu YY, Zhan WW, Li DH, Wang Y, Xiao Q, Liu J, Chen SD (2016) Substantia nigra echogenicity correlated with clinical features of Parkinson's disease. Parkinsonism Relat Disord 24:28-33

9. Aarsland D, Larsen JP, Cummins JL, Laake K (1999) Prevalence and clinical correlates of psychotic symptoms in Parkinson disease: a community-based study. Arch Neurol 56:595-601

10. Goetz CG, Stebbins GT, Ouyang B (2011) Visual plus nonvisual hallucinations in Parkinson's disease: development and evolution over 10 years. Mov Disord 26:2196-2200

11. Gibson G, Mottram PG, Burn DJ, Hindle JV, Landau S, Samuel M, Hurt CS, Brown RG, M Wilson KC (2013) Frequency, prevalence, incidence and risk factors associated with visual hallucinations in a sample of patients with Parkinson's disease: a longitudinal 4-year study. Int J Geriatr Psych 28:626-631

12. McKeith IG, Dickson DW, Lowe J, Emre M, O'Brien JT, Feldman H, Cummings J, Duda JE, Lippa C, Perry EK, Aarsland D, Arai H, Ballard CG, Boeve B, Burn DJ, Costa D, del Ser T, Dubois B, Galasko D, Gauthier S, Goetz CG, Gomez-Tortosa E, Halliday G, Hansen LA, Hardy J, Iwatsubo T, Kalaria RN, Kaufer D, Kenny RA, Korczyn A, Kosaka K, Lee VM, Lees A, Litvan I, Londos E, Lopez OL, Minoshima S, Mizuno Y, Molina JA, MukaetovaLadinska EB, Pasquier F, Perry RH, Schulz JB, Trojanowski JQ, Yamada M, Consortium on DLB (2005) Diagnosis and management of dementia with Lewy bodies: third report of the DLB Consortium. Neurology 65:1863-1872

13. Fahn S, Elton RL (1987) Unified Parkinson's disease rating scale. In: vol., pp. 153-163,293-304. Recent Developments in Parkinsons Disease, Vol 2 Florham Park, NJ: Macmillan Healthcare Information.

14. Martinez-Martin P, Rodriguez-Blazquez C, Abe K, Bhattacharyya KB, Bloem BR, Carod-Artal FJ, Prakash R, Esselink RA, FalupPecurariu C, Gallardo M, Mir P, Naidu Y, Nicoletti A, Sethi K, Tsuboi Y, van Hilten J, Visser M, Zappia M, Chaudhuri KR (2009) International study on the psychometric attributes of the non-motor symptoms scale in Parkinson disease. Neurology 73: 1584-1591

15. Chaudhuri KR, Pal S, DiMarco A, Whately-Smith C, Bridgman K, Mathew R, Pezzela FR, Forbes A et al (2002) The Parkinson's disease sleep scale: a new instrument for assessing sleep and nocturnal disability in Parkinson's disease. J Neurol Neurosurg Psychiatry 73:629-635

16. Varma MG, Wang JY, Berian JR, Patterson TR, McCrea GL, Hart SL (2008) The constipation severity instrument: a validated measure. Dis Colon Rectum 51:162-172

17. Nilsson MH, Bladh S, Hagell P (2013) Fatigue in Parkinson's disease: measurement properties of a generic and a condition-specific rating scale. J Pain Symptom Manag 46:737-746

18. Folstein MF, Folstein SE, McHugh PR (1975) Mini-mental state. A practical method for grading the cognitive state of patients for the clinician J Psychiatr Res 12:189-198

19. Hamilton M (1960) A rating scale for depression. J Neurol Neurosurg Psychiatry 23:56-62

20. Hamilton M (1959) The assessment of anxiety states by rating. Br J Med Psychol 32:50-55

21. Peto V, Jenkinson C, Fitzpatrick R, Greenhall R (1995) The development and validation of a short measure of functioning and well being for individuals with Parkinson's disease. Qual Life Res Int J Qual Life Asp Treat Care Rehab 4:241-248 
22. Emre M, Aarsland D, Brown R, Burn DJ, Duyckaerts C, Mizuno Y, Broe GA, Cummings J et al (2007) Clinical diagnostic criteria for dementia associated with Parkinson's disease. Mov Disord 22: 1689-1707 quiz 1837

23. Wan Y, Wang G, Zeng J, Laake K (2009) Investigation on prevalence and risk factors of visual hallucination in patients with Parkinson's disease. J Shanghai Jiaotong Univ 29:862-865

24. Meppelink AM, de Jong BM, Renken R, Leenders KL, Cornelissen FW, van Laar T (2009), Impaired visual processing preceding image recognition in Parkinson's disease patients with visual hallucinations. Brain: a journal of neurology 132:2980-2993.

25. Ibarretxe-Bilbao N, Ramirez-Ruiz B, Junque C, Marti MJ, Valldeoriola F, Bargallo N, Juanes S, Tolosa E (2010) Differential progression of brain atrophy in Parkinson's disease with and without visual hallucinations. J Neurol Neurosurg Psychiatry 81:650-657

26. Lenka A, Jhunjhunwala KR, Saini J, Pal PK (2015) Structural and functional neuroimaging in patients with Parkinson's disease and visual hallucinations: a critical review. Parkinsonism Relat Disord 21:683-691

27. Ward RJ, Zucca FA, Duyn JH, Crichton RR, Zecca L (2014) The role of iron in brain ageing and neurodegenerative disorders. The Lancet Neurology 13:1045-1060

28. Berg D, Grote C, Rausch WD, Maurer M, Wesemann W, Riederer $P$, Becker $G$ (1999) Iron accumulation in the substantia nigra in rats visualized by ultrasound. Ultrasound Med Biol 25:901-904

29. Berg D, Roggendorf W, Schroder U, Klein R, Tatschner T, Benz P, Tucha O, Preier M et al (2002) Echogenicity of the substantia nigra: association with increased iron content and marker for susceptibility to nigrostriatal injury. Arch Neurol 59:999-1005

30. Tuite PJ, Provias JP, Lang AE (1996) Atypical dopa responsive parkinsonism in a patient with megalencephaly, midbrain Lewy body disease, and some pathological features of HallervordenSpatz disease. J Neurol Neurosurg Psychiatry 61:523-527

31. Berg D, Godau J, Riederer P, Gerlach M, Arzberger T (2010), Microglia activation is related to substantia nigra echogenicity. Journal of neural transmission (Vienna, Austria : 1996) 117: 1287-1292.

32. Imamura K, Hishikawa N, Sawada M, Nagatsu T, Yoshida M, Hashizume Y (2003) Distribution of major histocompatibility complex class II-positive microglia and cytokine profile of Parkinson's disease brains. Acta Neuropathol 106:518-526

33. Zhu Y, Wang B, Tao K, Yang H, Wang Y, Zhou T, Yang Y, Yuan L et al (2017) Iron accumulation and microglia activation contribute to substantia nigra hyperechogenicity in the 6-OHDA-induced rat model of Parkinson's disease. Parkinsonism Relat Disord 36:76-82

34. Sakai K, Ikeda T, Ishida C, Komai K, Yamada M (2019), Delusions and visual hallucinations in a patient with Parkinson's disease with dementia showing pronounced Lewy body pathology in the nucleus basalis of Meynert. Neuropathology: official journal of the Japanese Society of Neuropathology 39:319-323.

35. Zhu J, Shen B, Lu L, Lan W, Pan Y, Zhang L, Dong J, Wang M et al (2017) Prevalence and risk factors for visual hallucinations in Chinese patients with Parkinson's disease. J Neurol Sci 372:471476

36. Trojano L, Papagno C (2018), Cognitive and behavioral disorders in Parkinson's disease: an update. II: behavioral disorders. Neurological sciences: official journal of the Italian Neurological Society and of the Italian Society of Clinical Neurophysiology 39:53-61.

37. Walter U, Skoloudik D, Berg D (2010) Transcranial sonography findings related to non-motor features of Parkinson's disease. J Neurol Sci 289:123-127

38. Berg D, Siefker C, Becker G (2001) Echogenicity of the substantia nigra in Parkinson's disease and its relation to clinical findings. $\mathrm{J}$ Neurol 248:684-689

39. Kim JY, Kim ST, Jeon SH, Lee WY (2007), Midbrain transcranial sonography in Korean patients with Parkinson's disease. Movement disorders: official journal of the Movement Disorder Society 22:1922-1926.

40. Luo WF, Zhang YC, Sheng YJ, Fang JC, Liu CF (2012), Transcranial sonography on Parkinson's disease and essential tremor in a Chinese population. Neurological sciences: official journal of the Italian Neurological Society and of the Italian Society of Clinical Neurophysiology 33:1005-1009.

41. Hagenah J, Konig IR, Sperner J, Wessel L, Seidel G, Condefer K, Saunders-Pullman R, Klein C et al (2010) Life-long increase of substantia nigra hyperechogenicity in transcranial sonography. NeuroImage 51:28-32

42. Bor-Seng-Shu E, Pedroso JL, Felicio AC, de Andrade DC, Teixeira MJ, Braga-Neto P, Batista IR, Barsottini OG, Borges V, Ferraz HB, Shih MC, Bressan RA, de Andrade LA, Walter U (2014) Substantia nigra echogenicity and imaging of striatal dopamine transporters in Parkinson's disease: a cross-sectional study. Parkinsonism Relat Disord 20:477-481

43. Mahlknecht P, Stockner H, Kiechl S, Willeit J, Rastner V, Gasperi A, Rungger G, Poewe W, et al. (2012), Is transcranial sonography useful to distinguish drug-induced parkinsonism from Parkinson's disease? Movement disorders: official journal of the Movement Disorder Society 27:1194-1196.

44. Hershkovitz I, Greenwald C, Rothschild BM, Latimer B, Dutour O, Jellema LM, Wish-Baratz S (1999) Hyperostosis frontalis interna: an anthropological perspective. Am J Phys Anthropol 109:303-325

Publisher's note Springer Nature remains neutral with regard to jurisdictional claims in published maps and institutional affiliations. 